Research Paper

\title{
Age and Gender Are Important Considerations in Choosing the Sniffing Position for Laryngoscopic View
}

\author{
Sung-Wook Park ${ }^{1}$, Kang-Woo Lee ${ }^{1}$, Myung-Soo Jang ${ }^{1}$, Jun-Young Jung², Bong-Jae Lee², Jong-Man Kang ${ }^{2}$ \\ 1. Department of Anesthesiology and Pain Medicine, Kyung Hee University Hospital, Kyung Hee University, Seoul, Korea, \\ 2. Department of Anesthesiology and Pain Medicine, Kyung Hee University Hospital at Gangdong, Kyung Hee University, Seoul, Korea
}

$\triangle$ Corresponding author: Jong-Man Kang, M.D., address: Department of Anesthesiology, Kyung Hee University Hospital at Gangdong, 149 Sangil-dong Gangdong-Gu, Seoul, Korea. Zip code: 134-090. Tel: 82-2-440-6193; Fax: 82-2-440-7808; E-mail: kjm@khnmc.or.kr

( ) Ivyspring International Publisher. This is an open-access article distributed under the terms of the Creative Commons License (http://creativecommons.org/ licenses/by-nc-nd/3.0/). Reproduction is permitted for personal, noncommercial use, provided that the article is in whole, unmodified, and properly cited.

Received: 2014.01.14; Accepted: 2014.09.1I; Published: 2014.10.02

\begin{abstract}
Aims: The sniffing position is considered to be the standard position for direct laryngoscopic viewing. This crossover study evaluated age and gender as variables in comparing the benefits of the sniffing position over simple head extension for laryngeal view during direct laryngoscopy.

Methods: Laryngoscopy with a curved blade was performed on 200 anesthetized adults (100 males, 100 females) presenting for routine elective surgery. Glottic visualization was assessed by using the percentage of glottic opening (POGO) score in both simple extension and sniffing positions without the aid of the assistant or external laryngeal manipulation. Each gender group was divided into a younger group ( $<50$ years) and an older group ( $\geq 50$ years). POGO scores were compared between both positions within each group.

Results: Mean (SD) POGO scores increased significantly only in younger male patients from $43 \%$ (39\%) in the head extension position to $76 \%(30 \%)$ in the sniffing position.

Conclusion: The sniffing position seems to be advantageous for getting a better laryngeal view during laryngoscopy for tracheal intubation in adult male patients less than 50 years old.
\end{abstract}

Key words: age, gender, POGO, head extension, laryngeal view, sniffing position

\section{Introduction}

A good laryngeal view is preferable for a successful tracheal intubation. The sniffing position has been thought to be the standard position for direct laryngoscopic view. However, Adnet et al. [1] showed no significant advantage of the sniffing position over simple head extension on routine general surgical patients, using the Cormack-Lehane scale. They found the sniffing position to be superior only in patients who were obese and in those with limited head extension. On the other hand, Levitan et al. [2] demonstrated significantly improved scores for percentage of glottic opening (POGO) on 7 fresh cadavers by using the head-elevated position. However, evaluation of the laryngeal view can be influenced by the grading method being used. The POGO scoring system provides objective, numerical, and continuous data compared with the traditional Cormack scale [3].
There have been no studies done on the effect of the sniffing position during laryngoscopy on patients according to gender or age, using POGO scores.

We hypothesized that age and gender could influence the relationship of the oral, pharyngeal, and laryngeal axes and that these differences would make the effect that the sniffing position has on the laryngeal view during tracheal intubation uneven between young and old or men and women.

The purpose of this study was to evaluate the effects of age and gender on the advantage of the sniffing position compared with simple head extension for laryngeal view during direct laryngoscopy, using POGO score.

\section{Materials and methods}

The study was approved by the Institutional 
Review Board of the Kyung Hee University Hospital (ref: KHNMC IRB 2012-074) and registered with Clinical Trials (ref: NCT01688661). Written and informed consent was obtained from each patient. Two hundred adults (100 males, 100 females, American Society of Anesthesiologists Class 1 or 2) presenting for routine elective surgery under general anesthesia were studied from August 2012 to March 2013. Patients with anatomical defects of the face, neck, or upper airway were excluded from the study.

The following properties were evaluated and recorded: (1) mouth opening, as the interincisor gap with the mouth fully opened, (2) thyromental distance along a straight line from the thyroid notch to the lower border of the mandibular mentum with the head in full extension, and (3) modified Mallampati classification.

Patients were placed on the operating table with the head in the simple extension position. Routine monitors were used. Induction of anesthesia was achieved with $1.5 \mathrm{mg} \mathrm{kg}^{-1}$ propofol IV. Muscle relaxation was obtained with $0.6 \mathrm{mg} \mathrm{kg}^{-1}$ rocuronium IV. Anesthesia was maintained with sevoflurane (2-3\%). After loss of all four twitches on the train-of-four obtained by ulnar nerve stimulation, laryngoscopy was performed using a Macintosh blade size 3. Each study subject was laryngoscoped twice - once in the simple extension position and then in the sniffing position, successively. All three anesthesiologists participating in the study had at least 3 years of experience in clinical anesthesia and had performed at least 1000 tracheal intubations in patients. The table height was set to the lowest setting because the heights of all three anesthesiologists were similar, between $172 \sim 176 \mathrm{~cm}$, and the stances used by the anesthetists were as comfortable and casual as feet shoulder-width apart, knees slightly bent. Glottic visualization was assessed

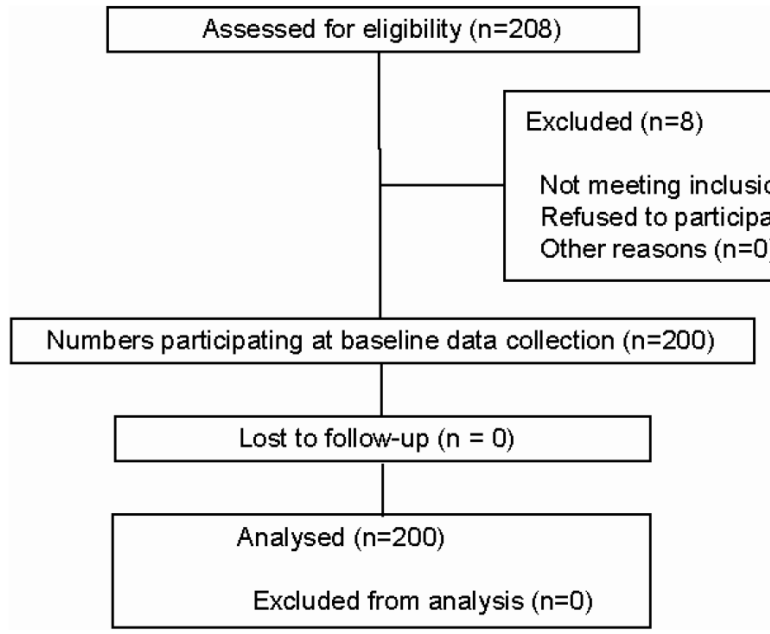

Figure I. Flow diagram. by using the POGO score, without any aid of assistant or external laryngeal manipulation in both the simple extension and sniffing positions. The sniffing position was achieved by insertion of a rigid box $(7 \mathrm{~cm}$ in height) under the head. We used a $7-\mathrm{cm}$ high rigid pillow to avoid the variable degree of cervical flexion caused by the compressibility of a soft head support [4]. The POGO score describes how much of the glottic opening is visible; a $100 \%$ POGO score includes visualization of the entire glottic opening from the anterior commissure of the vocal cords to the interarytenoid notch. A POGO score of $0 \%$ corresponds with no visualization of glottis, whether the epiglottis is seen or not [3].

Assuming a clinically significant difference in POGO score of $25 \%$ [5], we calculated the appropriate sample size to be 18 patients with use of $\alpha=0.05$ and $\beta$ $=0.9$. We recruited 100 patients of each gender for the study. Secondarily, each gender group was divided into a younger group ( $<50$ years) and an older group ( $\geq 50$ years), and patients were recruited until each subgroup reached 50 patients, for general consistency, based on the central limit theory despite the necessary sample size being calculated as 18 . POGO scores were compared between both positions within each group. The percentage of cases showing improvement or decline (significant change: more than 25\% POGO) in the sniffing position compared to the simple head extension position was also noted in each group.

Independent t-test or Fisher's exact test was used for statistical analysis comparing the characteristics between the gender groups or the age groups. POGO scores of each patient in both positions were analyzed by paired Student's t-test using SPSS statistical software (version 15.0). Chi square analysis was used to determine improved or diminished cases between the gender groups and age groups. $\mathrm{P}<0.05$ was considered statistically significant.

\section{Results}

Of the 208 patients assessed, 200 were enrolled in the study and progressed throughout the study (Figure 1).

Patient characteristics are shown in Table 1. The POGO scores increased only in the younger male patients from $43 \%$ (SD: $39 \%$ ) in the simple head extension position to $76 \%(30 \%)$ in the sniffing position (Table 2). In half of the younger male patients, POGO scores increased meaningfully (more than 25\%) in the sniffing position (Table 3 ). 
Table I. Baseline Characteristics of Patients.

\begin{tabular}{|c|c|c|c|c|}
\hline & \multicolumn{2}{|c|}{ Males $(n=100)$} & \multicolumn{2}{|c|}{ Females $(n=100)$} \\
\hline & $\begin{array}{l}\text { Younger } \\
(\mathrm{n}=50)\end{array}$ & $\begin{array}{l}\text { Older } \\
(\mathrm{n}=50)\end{array}$ & $\begin{array}{l}\text { Younger } \\
(\mathrm{n}=50)\end{array}$ & $\begin{array}{l}\text { Older } \\
(\mathrm{n}=50)\end{array}$ \\
\hline \multirow[t]{2}{*}{ Age (yrs) } & $46(21-75)$ & & $47(18-73)$ & \\
\hline & $34(21-48)$ & $61(50-75)$ & $36(18-48)$ & $59(50-73)$ \\
\hline Height (cm) & $172 \pm 5$ & & $157 \pm 6^{*}$ & \\
\hline Weight (kg) & $71 \pm 11$ & & $60 \pm 9^{*}$ & \\
\hline $\begin{array}{l}\text { Mouth opening }<35 \\
\mathrm{~mm}(\%)\end{array}$ & 0 & & 3 & \\
\hline $\begin{array}{l}\text { Thyromental distance } \\
<60 \mathrm{~mm}(\%)\end{array}$ & 0 & & 2 & \\
\hline $\begin{array}{l}\text { Mallampati class } \\
\text { III-IV (\%) }\end{array}$ & 14 & & 4 & \\
\hline
\end{tabular}

Table 2. Mean POGO scores at two different positions.

\begin{tabular}{lll}
\hline & $\begin{array}{l}\text { POGO (head extension } \\
\text { position) }\end{array}$ & POGO (sniffing position) \\
\hline Males $(\mathrm{n}=100)$ & $42 \pm 39(0 \sim 100), 22$ & $58 \pm 35^{*}(0 \sim 100), 3$ \\
Younger $(\mathrm{n}=50)$ & $43 \pm 39(0 \sim 100), 10$ & $76 \pm 30^{*}(0 \sim 100), 1$ \\
Older $(\mathrm{n}=50)$ & $41 \pm 39(0 \sim 100), 12$ & $40 \pm 33(0 \sim 100), 2$ \\
Females $(\mathrm{n}=100)$ & $45 \pm 33(0 \sim 100), 11$ & $47 \pm 30(0 \sim 100), 10$ \\
Younger $(\mathrm{n}=50)$ & $50 \pm 33(0 \sim 100), 2$ & $52 \pm 32(0 \sim 100), 4$ \\
Older $(\mathrm{n}=50)$ & $40 \pm 32(0 \sim 100), 9$ & $41 \pm 29(0 \sim 100), 6$
\end{tabular}

*Significantly different $(p<0.05)$ between the positions. Data shown are mean $\pm \mathrm{SD}$ (range), number of patients who had a POGO score of $0 \%$.

Table 3. The number of patients showing improvement or decline (significant change of more than $25 \%$ in POGO score) in the sniffing position compared to the simple head extension position.

\begin{tabular}{lll}
\hline & Improved & Declined \\
\hline Males $(\mathrm{n}=100)$ & $31^{*}$ & 7 \\
Younger $(\mathrm{n}=50)$ & $26^{*},+$ & 0 \\
Older $(\mathrm{n}=50)$ & 5 & 7 \\
Females $(\mathrm{n}=100)$ & 10 & 7 \\
Younger $(\mathrm{n}=50)$ & 6 & 2 \\
Older $(\mathrm{n}=50)$ & 4 & 5 \\
\hline
\end{tabular}

*Significantly different $(p<0.05)$ between the gender groups. †Significantly different $(p<0.05)$ between the age groups.

\section{Discussion}

The theoretical background of the sniffing position is associated with the alignment of the oral, pharyngeal, and laryngeal axes. The mutual relationship of head and neck in the supine position can vary depending on the difference of physical status, weight, height, cervical curvature, the length of neck, head size, and so on. This craniocervical posture can affect the alignment of the three axes and then influence the effect of the sniffing position on glottic visualization.

There are two groups of obstacles between the eyes of the laryngoscopist and the vocal cords. The first is the upper teeth, maxilla, and other anatomical features located posterior to the oral airway space. The second is the tongue, epiglottis, mandible, and other anatomical features located anterior to the airway. The sniffing position is obtained by two consecutive movements, anterior flexion of the lower cervical spine (neck flexion) and extension of the atlanto-occipital joint (head extension). Each movement has its own role in displacement of the obstacles for better visualization of the vocal cords by the laryngoscopist. Neck flexion produces an upward movement of both groups of obstacles and head extension moves the posterior obstacles downward [6].

This study showed that the sniffing position offers a great advantage only in younger male patients, meaning that the sniffing position provides an optimal relation of neck flexion and head extension in younger male patients. It is not clear why the sniffing position was not superior to the simple extension position in female patients and older male patients. However, the disparity may be assumed to be associated with the differences in the size and flexibility of the head and neck. For most anthropometric parameters including head height, neck length, and pharyngeal airway length, females are significantly smaller than males $[7,8]$. Therefore, a hard pillow of $7-\mathrm{cm}$ height may be too high to obtain appropriate neck flexion and head extension simultaneously in the female. From radiographic data of 1,200 asymptomatic subjects, it was established that cervical lordosis develops with aging, while the height of the vertebral body and range of cervical motion, especially extension, decrease gradually [9]. Moreover, restricted craniocervical extension can impose a severe restraint on mouth opening, one of the crucial factors to approach the airway [10]. Levitan et al. [11] described the ideal head positioning for laryngoscopy of an adult patient as slight head elevation (neck flexion) and extreme atlanto-occipital extension by elevating the patient's head 8 to $10 \mathrm{~cm}$ and tilting the head backwards as far as possible. However, neck flexion itself in the sniffing position can restrain atlanto-occipital extension to some degree. Moreover, atlanto-occipital extension can be partially counteracted by lifting the laryngoscope with an upward and forward motion after insertion of the laryngoscope blade during direct laryngoscopy if there is not any support for the patient's head to maintain the state of atlanto-occipital extension. This phenomenon can be worse in the sniffing position using a hard pillow rather than in the simple extension position. On the other hand, a simple extension position itself can even produce some neck flexion because the lower neck and the occiput remain in contact with the surface [12]. From these points of view, the sniffing position can be superior to the simple head extension position only if atlanto-occipital extension is maintained maximally. We think that these conditions can be met only in a 
younger male patient with his head placed on a 7-cm height hard pillow.

Differences in POGO scores of $25 \%$ are associated with an increased number of laryngoscopies required to perform successful tracheal intubation [13]. In $50 \%$ of younger male patients, the POGO scores improved significantly, exclusive of the rest of the study participants. It may be impossible or probably unnecessary to predict which position would be beneficial for each individual since determining whether the sniffing or simple extension position is advantageous to getting a better laryngeal view during direct laryngoscopy depends on the physical condition of the patient. If the glottic visualization is not good in one position, the other position can be tried subsequently. However, we can recommend that the sniffing position should be the first choice in adult male patients, especially those of younger age.

This study has several limitations. We could not technically blind the laryngoscopist to the position of the patient. The laryngoscopist tried to get the maximal laryngeal view in each position to reduce the potential bias of a non-blind study design. POGO scoring depends only on the amount of glottic opening. A zero score for POGO means a difficult laryngoscopy, including the Cormack grades III and IV. It can be considered as a weakness of POGO scoring that it does not have any score below zero. However, we chose POGO scoring over Cormack and Lehane grades due to its high validation and reliability. The intraobserver correlation coefficient of POGO scoring is 0.85 , while the interobserver correlation is 0.74 . The intrarater reliability of POGO scoring seems to be as good as or better than that of Cormack and Lehane grades. Moreover, the POGO score can detect a subtle change of laryngeal view between various intubation techniques and then provide better outcomes for comparing the techniques [3].

In conclusion, the sniffing position seems to be advantageous to getting a better laryngeal view during laryngoscopy for tracheal intubation, especially in adult male patients younger than 50 years old.

\section{Competing Interests}

The authors have declared that no competing interest exists.

\section{References}

1. Adnet F, Baillard C, Borron SW, Denantes C, Lefebvre L, Galinski M, Martinez C, Cupa M, Lapostolle F. Randomized study comparing the "sniffing position" with simple head extension for laryngoscopic view in elective surgery patients. Anesthesiology 2001; 95: 836-41.

2. Levitan RM, Mechem CC, Ochroch EA, Shofer FS, Hollander JE. Head-elevated laryngoscopy position: improving laryngeal exposure during laryngoscopy by increasing head elevation. Ann Emerg Med 2003; 41: 322-30.

3. Levitan RM, Ochroch EA, Kush S, Shofer FS, Hollander JE. Assessment of airway visualization: validation of the percentage of glottic opening (POGO) scale. Acad Emerg Med 1998; 5: 919-23.
4. Takenaka I, Aoyama K, Iwagaki T, Ishimura H, Kadoya T. The sniffing position provides greater occipito-atlanto-axial angulation than simple head extension: a radiological study. Can J Anaesth 2007; 54: 129-33.

5. Lee BJ, Kang JM, Kim DO. Laryngeal exposure during laryngoscopy is better in the 25 degrees back-up position than in the supine position. Br J Anaesth 2007; 99: 581-6.

6. Isono S. Common practice and concepts in anesthesia: time for reassessment: is the sniffing position a "gold standard" for laryngoscopy? Anesthesiology 2001; 95: 825-7.

7. Vasavada AN, Danaraj J, Siegmund GP. Head and neck anthropometry, vertebral geometry and neck strength in height-matched men and women. J Biomech 2008; 41: 114-21.

8. Shigeta Y, Ogawa T, Venturin J, Nguyen M, Clark GT, Enciso R. Gender- and age-based differences in computerized tomographic measurements of the orophaynx. Oral Surg Oral Med Oral Pathol Oral Radiol Endod 2008; 106: 563-70.

9. Yukawa Y, Kato F, Suda K, Yamagata M, Ueta T. Age-related changes in osseous anatomy, alignment, and range of motion of the cervical spine. Part I: Radiographic data from over 1,200 asymptomatic subjects. Eur Spine J 2012:

10. Calder I, Picard J, Chapman M, O'Sullivan C, Crockard HA. Mouth opening: a new angle. Anesthesiology 2003; 99: 799-801.

11. Levitan R, Ochroch EA. Airway management and direct laryngoscopy. A review and update. Crit Care Clin 2000; 16: 373-88.

12. Adnet F, Borron SW, Dumas JL, Lapostolle F, Cupa M, Lapandry C. Study of the "sniffing position" by magnetic resonance imaging. Anesthesiology 2001; 94: 83-6.

13. Ochroch AK HJ, Levitan RM. POGO score as a predictor of intubation difficulty and need for rescue devices [abstract]. Ann Emerg Med 2000; 36: A199. 